\title{
Editorial
}

\section{Adenocarcinoma of the esophago-gastric junction}

\author{
J.R. SIEWERT \\ Department of Surgery, Technische Universität München, Ismaninger Strasse 22 D-81675, Munich, Germany
}

There are at least two reasons for intensive clinical and scientific research concerning adeno-carcinoma of the esophago-gastric junction (AEG):

- first, the incidence of this tumor entity is rising dramatically in the Western world;

- second, according to the current literature, the prognosis of this disease is still very poor.

Therefore, this tumor entity comes more and more into the focus of clinicians and scientists. These tumors need special and specialized surgical care. Therapeutical guidelines need to be investigated.

The study of the current literature demonstrates that the tumors of the esophago-gastric junction are insufficiently defined. This tumor entity encompasses variable and anatomically different tumors. There are only a few articles concerning the treatment of these tumors, and they do not agree on an exact definition. A typical example is the controlled study by Walsh et al. [1], but results are weakened by this obstacle. Furthermore, a comparison between treatment results is hardly possible.

Using the definition and classification of AEG by our group [2], this problem can be solved. The definition includes adenocarcinomas in the region $5 \mathrm{~cm}$ above and $5 \mathrm{~cm}$ below the anatomical cardia (the transition of the two-layered muscle wall of the esophagus to the threelayered muscle of the stomach). In this region, three different tumor entities arise:

- adenocarcinoma of the distal esophagus (AEG I, Barrett);

- "real" carcinoma of the cardia (AEG II);

- subcardial carcinoma of the stomach, infiltrating the distal esophagus (AEG III).

The analysis of our own patient population with these tumors $(n=817)$ demonstrates that especially the distal Barrett carcinoma is a tumor entity on its own. It has to be distinguished from AEG types II and III and further from squamous cell carcinoma of the esophagus. There are also great differences concerning the socioeconomic characteristics of the patients.

This analysis was the prerequisite to establish distinct therapeutical concepts. These different entities need different surgical approaches:

- AEG I is treated by transhiatal radical esophagectomy;

- AEG II and III tumors are treated by transhiatal extended gastrectomy.

Since the consensus conference of the IGCA and ISDE in 1997, this classification is being accepted more and more internationally. This will hopefully lead to more clarity concerning the treatment modalities [3-5]. On the basis of this definition, the aim is to evaluate different therapeutical concepts. In our own patient population a significant improvement in prognosis could be achieved by new standardized therapeutical concepts, which are based on the definition mentioned above.

The work of Peracchia et al. [5] is a major contribution in this context. It has been demonstrated that there is still a need for discussion concerning different treatment and classification (treatment as esophageal cancer or as gastric cancer?) of AEG II tumors. Only on the basis of this clarity in classification will transparency be achieved in the future.

For the time being the issue is still open. For instance, discussions are ongoing regarding the pathogenesis of type II cancer as a possibly reflux-induced malignant degeneration via so-called carditis.

This interpretation would be close to the oncogenesis of type I cancer. On the other hand, the investigation of molecular markers-mutations of the $p 53$ gene are much more frequently seen in type I than in type II and type III cancers-brings real cardia cancer much closer to gastric cancer. Of clinical relevance is the discussion about the therapeutical consequences. In our own 
experience the prognosis of type II cancer following an adequate enlarged total gastrectomy ( $\mathrm{R} 0$ resection) is as good as that following a subtotal esophagectomy and proximal gastrectomy. The subtotal esophagectomy has on the other hand a higher morbidity and mortality in comparison with the enlarged total gastrectomy. These are the reasons we have decided to treat type II cancer as gastric cancer. But on this point other conclusions can also be drawn [6]. Once more a clear classification of AEG builds the fundament of a fruitful discussion.

\section{References}

1. Walsh TN, Noonan N, Hollywood D, Kelly A, Keeling N, Hennessy TPJ. A comparison of multimodal therapy and surgery for esophageal adenocarcinoma. New Engl J Med 1996;335:462-7.
2. Siewert JR, Stein HJ. Classification of adenocarcinoma of the esophagogastric junction. Br J Surg 1998;85:1457-9.

3. Dresner SM, Wayman J, Bennett MK, Hayes N, Griffin SM. Pattern of lymph node dissemination from adenocarcinoma of the oesophagogastric junction [abstract]. Br J Surg 1999;86:424.

4. Fein M, Fuchs KH, Ritter MP, Freys SM, Heimbucher A, Staab C, et al. Application of the new classification for cancer of the cardia. Surgery 1998;124:707-14.

5. Peracchia A, Bonavina L, Incarbone R. Results of surgical therapy in patients with adenocarcinoma of the esophagus and cardia. Gastric Cancer 1999;2:89-94.

6. Wijnhoven BPL, Siersema PD, Hop WCJ, van Dekken H, Tilanus HW on behalf of the Rotterdam Oesophageal Tumour Study Group. Adenocarcinomas of the distal oesophagus and gastric cardia are one clinical entity. Br J Surg 1999;86:529-35. 